\title{
Frequency, Epidemiology and Outcome of Acute Kidney Injury Among Patients Admitted to Nephrology Center, Sana'a: Observational Study
}

\author{
Nagib Wazae Abuasba ${ }^{1}$, Khalid Alwadee ${ }^{2}$, Mushtaq Hasan Al-Hamadi ${ }^{2}$ \\ ${ }^{1}$ Nephrology Department, Faculty of Medicine, Sana'a University, Sana'a, Yemen \\ ${ }^{2}$ Nephrology Department, Al Thawra Hospital, Sana'a, Yemen
}

Email address:

dr.khalidalwadei $a$ gmail.com (K. Alwadee), mushtaq@yahoo.com (M. H. Al-Hamadi)

To cite this article:

Nagib Wazae Abuasba, Khalid Alwadee, Mushtaq Hasan Al-Hamadi. Frequency, Epidemiology and Outcome of Acute Kidney Injury Among Patients Admitted to Nephrology Center, Sana'a: Observational Study. American Journal of Internal Medicine. Vol. 6, No. 5, 2018, pp. 121-125. doi: 10.11648/j.ajim.20180605.15

Received: July 11, 2018; Accepted: July 24, 2018; Published: September 3, 2018

\begin{abstract}
Although acute kidney injury (AKI) in our setting is growing rapidly, the available data on the magnitude of this lethal problem are very limited. The objective of this study was to determine the incidence, epidemiology and outcome of AKI. A total of 143 hospitalized patients with AKI in Al Thawra General Hospital, nephrology department between July 2015 and December 2016 were enrolled. The mean age of the study population was $37.7 \pm 8.4$ years, and $68.5 \%$ of these patients were males. Almost $81.8 \%$ of cases were medically referred and malarial infection was the commonest cause of AKI (23\%). Pre renal AKI was present in 65 patients $(45.4 \%)$ and renal AKI in 78 patients $(54.5 \%)$. Most cases were presented with clinical manifestation of volume overload $(65 \%)$, oliguria $(51.7 \%)$, anuria $(16.7 \%)$, and high serum creatinine level. The majority of cases $(58.7 \%)$ had recovered without dialysis. In-hospital mortality was observed in 11 patients $(7.7 \%)$. Hepatic failure and malarial infection were the common causes of death. This study demonstrates low frequency of AKI in our setting. The etiologies of this lethal health problem are preventable and treatable in approximately half of cases. Late referral to hospital may contribute both to the progression of renal disease and also to high mortality.
\end{abstract}

Keywords: Acute Kidney Injury, Yemen, In-Hospital Mortality

\section{Introduction}

Acute renal failure (ARF) is a serious complication in hospitalized patients. In the literature, there is more than 30 definitions of ARF leading to high variation in the reported incidence and outcome, for this reason the term acute kidney injury (AKI) has been recently used instead of ARF to provide a uniform definition and classification [1]. The new terminology enables healthcare professionals to consider the disease as a spectrum of injury extends from less severe forms to more advanced injury when AKI may require renal replacement therapy (RRT) [2]. AKI results in a significant burden for the society in terms of health resource use during the acute phase and the potential long-term complications including development of chronic kidney disease (CKD) and kidney failure [3]. AKI is characterized by an abrupt decrease in the renal function that occurs within hours or days, resulting in fluid and electrolytes imbalance [4].

It is responsible for approximately 2 million deaths annually worldwide [5] and the mortality rate reported among those patients with the most severe form of AKI requiring renal replacement therapy as $50-80 \%$ [6]. Several risk factors including aging, sepsis, cardiovascular and pulmonary regurgitation are associated with increased risk of AKI development [4].

Four in five cases of AKI occur in the developing countries and in these countries the risk of AKI varies between urban and rural areas, by season and cultural mores and according to the distribution of the infectious agents [3].

It is diagnosed and staged according to the level of serum creatinine as proposed by Kidney Disease Improving Global Outcome (KDIGO) [7].

In Yemen, there is no study using national data to determine the rate of AKI, etiology and mortality. 
Nephrology and urology center in Al Thawra general hospital, Sana'a is one of a few specialized centers in the country concerned with nephrology and urology management. It is equipped with modern facilities and capable to receive all cases from all areas. In our situation where most of the population density is concentrated away from the capital, spaced and diversity of the Yemeni's geography and the widespread of poverty make transfer patients to center more difficult. The aim of this study is to determine the incidence, epidemiology, and outcome of patients with AKI who admitted to the nephrology center in Al Thawra hospital.

\section{Methods and Materials}

This is a prospective observational study carried out between July 2015 and December 2016 at Al Thawra General Hospital, nephrology center. This study was approved by the Ethics committee of the hospital. Verbal consent was obtained from each participant. We included in this study all patients who admitted to the nephrology department with diagnosis of AKI. The diagnosis of AKI was based on detailed history, general examination and laboratory data. AKI was defined as a sudden deterioration of renal function presenting as oliguria (urine output $\leq 400 \mathrm{ml} /$ day) for at least 48 hours or as a rise in serum creatinine level of more than $50 \%$ and $\geq 2 \mathrm{mg} / \mathrm{dl}$. We excluded from this study all post renal AKI due to renal calculi because of its rarity as well as most cases are managed by urologists.

A data sheet was used for collecting the information of all patients including age, gender, residence area, coexisting morbidities, precipitating factors of AKI, acute hospitalrelated factors, duration of hospital stay, management and outcome. After admission, all investigations were performed including complete blood count (CBC), serum creatinine level, urea, liver function tests, electrolytes, urine analysis, and imaging studies. The management was followed as usual and dialysis was performed when indicated.

Pre renal failure was defined as when the causative factors (volume depletion) was rapidly corrected and renal functions return to normal.

Acute tubular necrosis was defined as when renal function did not improve after correction of a possible pre renal causes and when other interstitial, vascular, hepatic and glomerular causes were excluded.

All patients were followed up until discharge or death. Long - term follow - up is arranged depending on the severity of condition. Renal recovery was defined as when serum creatinine concentration returns to normal and partial recovery was defined as when serum creatinine concentration returned to above normal level at discharge. Results were analyzed using SPSS version 21. The incidence of AKI was defined as its occurrence as a proportion of the total admissions to the center. The results are expressed as frequencies or means \pm standard deviation. A $p$ value of $<$ 0.05 is considered statistically significant.

\section{Results}

A total of 143 patients were enrolled in this study over one and half year. The mean age on presentation was $37.7 \pm 8.4$ years, $68.5 \%$ were males and $31.4 \%$ females. The difference was statistically significant $(\mathrm{p}<0.001)$. Sana'a city was the most frequent referring area $38.4 \%$ followed by Ibb city $11.1 \%$ and Taiz city $9.7 \%$. Malaria was the most common cause of AKI accounted for $23 \%$, followed by gastroenteritis $12.5 \%$ and chronic kidney disease $10.4 \%$. Most cases were of medical causes $(81.8 \%)$, obstetrical $(11.1 \%)$ and only $6.9 \%$ of cases had surgical causes. Table 1 .

Table 1. Sociodemographic and clinical data of patients admitted with AKI.

\begin{tabular}{ll}
\hline Variable & N(\%) or mean \pm SD \\
\hline Total population $(\mathrm{n}=143)$ & $37.7 \pm 8.4$ \\
Age, year & \\
Gender & $98(68.5)$ \\
Male* & $45(31.4)$ \\
Female & \\
Referral area & $55(38.4)$ \\
Sana'a & $16(11.1)$ \\
Ibb & $14(9.7)$ \\
Taiz & $58(40.3)$ \\
Others & \\
Etiologic factor & $33(23)$ \\
Malarial infection & $18(12.5)$ \\
Gastroenteritis & $15(10.4)$ \\
Kidney disease & $16(11.1)$ \\
Obstetrical hemorrhage & $38(26.5)$ \\
Hypertension & $23(16)$ \\
Others & \\
Type of admission & $117(81.8)$ \\
Medical** & $16(11.1)$ \\
Obstetrical & $10(6.9)$ \\
Surgical &
\end{tabular}

$* \mathrm{X}^{2}=39.228, P<0.001, * * P<0.001$

The majority of cases (54.5\%) had acute tubular necrosis and $45,4 \%$ had prerenal AKI.. Volume overload (edema, raised JVP, crackles) was found among $65 \%$ including complicated volume overload like heart failure and/or pulmonary edema. Oliguria and anuria were found among $51.7 \%$ and $16.7 \%$ respectively. Neurological manifestations of uremia were reported in $10.4 \%$ of cases. More than $50 \%$ of patient admitted with serum creatinine level more than 600 $\mu \mathrm{mol} / \mathrm{l}$. The mean serum creatinine level was $819 \pm 39 \mu \mathrm{mol} / \mathrm{l}$. There were $58.7 \%$ of patients managed without dialysis while $33.5 \%$ needed dialysis with either peritoneal (PD) or hemodialysis (HD). The difference was statistically significant $(\mathrm{p}<0.001)$. The overall mortality rate was $7.7 \%$. Hepatic failure had the highest proportion of deaths $36.3 \%$, followed by malaria $27.2 \%$, pneumonia $18.1 \%$ and HELLP syndrome $18.1 \%$. They hospitalized for a mean of $13.4 \pm 5.7$ days. Table 2 .

Table 2. Clinical characteristics and outcome of patients with AKI.

\begin{tabular}{ll}
\hline Variable & N(\%) or mean \pm SD \\
\hline Type of AKI & \\
Pre renal & $65(45.4)$ \\
Renal & $78(54.5)$ \\
\hline
\end{tabular}




\begin{tabular}{ll}
\hline Variable & $\mathbf{N}(\%)$ or mean \pm SD \\
\hline Clinical presentation & \\
Volume overload* & $93(65)$ \\
Neurological manifestation of uremia & $15(10.4)$ \\
Oliguria & $74(51.7)$ \\
Anuria & $24(16.7)$ \\
Serum creatinine $(\mu \mathrm{mol} / \mathrm{l})$ & $819 \pm 39$ \\
Mean hospital stay, days & $13.43 \pm 5.7$ \\
Outcome & \\
Recovery without dialysis** & $84(58.7)$ \\
Recovery after transient dialysis & $48(33.5)$ \\
Death & $11(7.7)$ \\
Causes of death & \\
Hepatic failure & $4(36.3)$ \\
Malarial infection & $3(27.2)$ \\
Pneumonia & $2(18.1)$ \\
HELLP syndrome & $2(18.1)$ \\
\hline
\end{tabular}

$* \mathrm{X}^{2}=25.650, \mathrm{p}<0.001 ; * * \mathrm{X}^{2}=18.209, p<0.001$

\section{Discussion}

The current study showed that the incidence of AKI identified in our center is $3.7 \%$. It is reported that AKI occurs in approximately $7 \%$ of all hospitalized patients and in up to 36 to $67 \%$ of critically ill patients depending on the definition used $[8,9]$. The rate of AKI has been growing at a rate of $14 \%$ per year in USA since 2001 [8]. In a population based approach, it is estimated the population incidence of AKI to be 2000 to 3000 per million people per year $[8,10]$. The lower incidence of AKI observed in this study could be explained by the small sample size of this study.

The reported increasing incidence of AKI among these studies is attributed to the improvement of diagnostic criteria for AKI and increasing awareness of health care providers. However, such increasing rate is alarming particularly in the low-income countries like ours, where the rate of infectious disease is rising and the facilities to manage patients as early as possible are unavailable. Given this, along with the evidence that the occurrence of even mild AKI is associated with a $50 \%$ higher risk of death [3], the situation appears dangerous and more efforts are required to combat this issue.

This study showed that the mean age of patients with AKI was 37.7 year and the most frequent age interval observed was from 31 - 35 years $(10.5 \%)$ followed by another peak at interval from $51-55$ years $(9.1 \%)$ and again over the age of 70 year (7\%). This figure is in agreement with another study which found the mean age was $42.64 \%$ years [2] and also in accordance to similar study done in India with mean age as $39.7 \%$ years [11]. AKI is strongly associated with older age which is likely related to the decline in the renal function among the elderly [12]. Age-related structural and functional differences in renal blood flow (RBF) and glomerular filtration rate (GFR) contribute significantly to susceptibility of the aged population to nephrotoxic response compared with adult [13]. However, there is evidence that AKI in the developing countries mostly affects individuals being generally younger than that of developing ones [14]. In our country, which is one of least developing countries, most cases of AKI are community-acquired with infection and obstetrical complications being responsible for the majority of cases.

Medical causes were the most common underlying process (81.8\%) followed by obstetrical causes of which the HELLP syndrome was responsible for a half of cases, followed by hemorrhage and preeclampsia. Only $6.9 \%$ of cases were having surgical causes. The medical etiologic factors of AKI in this study are similar to the pattern previously reported in the developing countries [14]. Infection with Falciparum malaria was the commonest cause of AKI followed by gastroenteritis, and kidney disease. It is reported that in severe malaria, the incidence of AKI varies widely and ranging from 20 to $60 \%$ [15]. Malaria in Yemen still a common infectious disease causing a wide range of complications including AKI especially in the northwest regions. It is therefore important to diagnose the underlying etiology as early as possible so that medical intervention can be effective prior to the development of renal injury.

Although gastroenteritis is a rare entity in the developed countries, still poses challenge in the developing ones due to poor hygienic condition, overcrowding and late referral to medical care [2].

The overall recovery rate in the present study was $92.3 \%$. Complete recovery was achieved in $58.7 \%$ of patients without renal replacement therapy while $33.5 \%$ of patients required transient dialysis that discontinued within 2-6 weeks after normalization of kidney function.

These finding are comparable to another study [4]. Prior study has reported rate of recovery as $66-90 \%$ [16]. It is found in the current study that only $8 \%$ of patients with pre renal AKI required dialysis while the remaining cases had acute tubular necrosis (ATN). In a study by Hickson et al [17], $73 \%$ of patients who recovered enough kidney function to discontinue dialysis did so by 3 months and $94 \%$ in 6 months after initiation of dialysis. However, there evidence that failure to achieve dialysis independent by 90 days would appear more likely to be associated with progression to the end stage renal disease [16].

In this study, it is found that the acute tubular necrosis was more frequent than pre renal disease which possibly reflects the delay in seeking care and therefore, late detection of AKI. A male predominance is observed in the current study, similar to another study [2]. It has been reported that AKI is strongly associated with male gender and black race [12]. Several studies have been evaluated whether the risk of AKI, severity, and progression to CKD are influencing by sex but the results are conflicting $[18,19]$. A recent study found that males are 2.2 times more likely to develop AKI requiring renal replacement therapy [18], conversely another study found the women are more likely to have hospital- acquired AKI $[7,20]$. There is evidence that the response to different pathological processes is different between male and female mediated by hormonal milieu [18, 19]. However, further study is needed to determine the mechanism of such differences. It is observed in this study that most patients admitted late to the hospital thus, the diagnosis of AKI was made rather late. It is not uncommon in our country that some patients may not look for medical care early because of 
poverty and traveling difficulties from rural areas until the disease becomes quite advanced. The overall mortality rate noted in this study was $7.7 \%$, and hepatic failure represented the main cause of death accounted for $36.3 \%$ followed by malarial infection (27.2\%), pneumonia (18.1\%) and HELLP syndrome $(18.1 \%)$.

It is reported in one study that in-hospital mortality rate of AKI was $29.6 \%$ [21]. Brown JR et al [8] in a study carried out in the USA from 2001 to 2011 found that the in-hospital mortality associated with AKI has been on the decline starting from $21.9 \%$ in 2001 to $9.1 \%$ in 2011 . The risk of death increased with the severity of AKI, and those requiring renal replacement therapy the death rate reaches to $60.3 \%$ [22]. There is a great variation in the mortality rates reported in the low-income regions compared with high-income countries, which could be related to several factors such as climate, geographical features, ethnic groups, cultures, socioeconomic status and developmental heterogeneity [23].

Thanchartwet $\mathrm{V}$ et al [24] found the mortality of AKI caused by Falciparum malaria as $14 \%$. They attributed the cause of death as multiorgan failure which included shock, cerebral malaria and metabolic acidosis. In our setting, it is difficult to estimate both the exact prevalence of AKI and also the mortality trend because of almost absence of data on this issue. However, more studies with a large national representative sample are urgently required. The mortality rate of AKI in the present study falls below the range quoted in the literature as 16 to $63.3 \%$ [21]. The likely explanation is the small sample size of this study. It is observed in the current study that the patient who developed neurological manifestation of uremia such as altered level of consciousness at admission, a high serum creatinine level, those required intensive care admission, and those needed dialysis were associated with higher in-hospital mortality. These findings have previously been strongly associated with increased mortality [25]. Although most death had been caused by the underlying disease rather than AKI itself, these factors should be considered strong predictors of death. Efforts therefore, should be focused on minimizing the causes of AKI through preventive strategies, as well as early recognition and management. It is clear that the general heath of patients and the degree of severity of the associated comorbidity makes a major contribution towards outcome. The present investigation found the mean hospital stay as $13.43 \pm 5.7$ days. The discharge practice of the patients with AKI in our center depends on the improvement of critical phase rather than complete normalization of the kidney function because the care of patients mostly extends beyond inpatient site and regular follow-up plan is arranged based on the severity of illness.

The study limitations are few, and the most notable one is that the long-term outcome could not be determined because the majority of patients were from rural settings so attending revisit checkup is very difficult.

Although this hospital-based study could not estimate the actual burden of the AKI in the country, it provides very important information that could be useful for further studies.

\section{Conclusion}

This study demonstrates the incidence of AKI is $3.7 \%$. The etiology of this serious health problem was found to be preventable and treatable in approximately half of cases. Patients who presented with neurological manifestation of uremia, higher serum creatinine level at admission, and those needed dialysis were associated with higher in-hospital mortality. Late referral to hospital may contribute both to the progression of renal disease and also to mortality.

\section{References}

[1] Krishnamurthy, S. Mondal N; Narayanan P; Biswal $\mathrm{N}$;Srinivasan S; Soundravally R et al. Incidence and etiology of acute kidney injury in southern India. Indian J. Pediatr 2013; 80, 183-189.

[2] Bhadade R, De'Souza R, Harde MJ, Mehta KS, Bhargava P. A Prospective Study of Acute Kidney Injury According to KDIGO Defi nition and its Mortality Predictors. J Assoc Physicians India. 2016; 64: 22- 28.

[3] Abd ElHafeez S, Tripepi G, Quinn R, Naga Y, Abdelmonem $\mathrm{S}$, Mohamed AbdelHady $\mathrm{M}$ et al. Risk, predictors, and outcomes of acute kidney injury in patients admitted to intensive care units inEgypt Scientific Reports | 7: 17163 | DOI:10.1038/s41598-017-17264-7

[4] Magden K, Yildirim I, Kutu M, Ozdemir M, Peynir S, Altas A, Yildiz G, Hur E. Recovery process in patients followedup due to acute kidney injury. Hippokratia 2013; 17(3):239242.

[5] Chawla, L. S. \& Kimmel, P. L. Acute kidney injury and chronic kidney disease: an integrated clinical syndrome. Kidney Int. 2012; 82, 516-524.

[6] Uchino S, Kellum JA, Bellomo R et al. Acute renal failure in critically ill patients: a multinational, multicenter study. JAMA 2005; 294: 813-818.

[7] Kidney Disease: Improving Global Outcomes (KDIGO). Acute Kidney Injury Work Group. KDIGO Clinical Practice Guideline for Acute Kidney Injury. Kidney Inter Suppl 2012; 2:1-138.

[8] Brown JR, Rezaee ME, Marshall EJ, Matheny ME. Hospital Mortality in the United States following Acute Kidney Injury. BioMed research international. 2016; 2016:4278579. [PMC free article] [PubMed]

[9] Dennen P, Douglas IS, Anderson R. Acute kidney injury in the intensive care unit: An update and primer for the intensivist. Crit Care Med 2010; 38:261-275.

[10] R. K. Hsu, C. E. McCulloch, R. A. Dudley, L. J. Lo, andC.-Y. Hsu, "Temporal changes in incidence of dialysis-requiring AKI," Journal of the American Society of Nephrology 2013; 24(1):37-42.

[11] Basu G, Chrispal A, Boorugu H, Gopinath KG, Chandy S, Prakash JA, et al. Acute kidney injury in tropical acute febrile illness in a tertiary care centre--RIFLE criteria validation. Nephrol Dial Transplant 2011; 26:524-31. 
[12] Jain A, McDonald HI, Nitsch D, Tomlinson L, Thomas SL. Risk factors for developing acute kidney injury in older people with diabetes and community-acquiredpneumonia: a population-based UK cohort study. BMC Nephrol 2017; 18(1): 142.

[13] KHAN, T. M.; KHAN, K. N. M. Acute kidney injury and chronic kidney disease. Veterinary Pathology, 2015; 52(3):441-444.

[14] Naicker S, Aboud O, Gharbi MB. Epidemiology of acute kidney injury in Africa. Semin Nephrol 2008; 28:348-53

[15] Saravu K, Rishikesh K, Parikh CR: Risk factors and outcomes stratified by severity of acute kidney injury in malaria. PLoS One 2014; $9: \mathrm{e} 90419$.

[16] Cerdá J, Liu KD, Cruz DN, Jaber BL, Koyner JL, Heung M, et al. Promoting Kidney Function Recovery in Patients with AKI Requiring RRT. Clin J Am Soc Nephrol. 2015; 10(10):1859-67. doi:10.2215/CJN.01170215.

[17] Hickson, L. J., Chadhary, S., Williams, A. W. et al. Predictors of outpatient kidney function recovery among patients who initiate hemodialysis in the hospital. Am J Kidney Dis. 2015; 65: 592-602.

[18] Neugarten J, Golestaneh L and Kolhe N V. Sex differences in acute kidney injury requiring dialysis. BMC Nephrology 2018; 19:131.

[19] Posada I L' Cortés C. P, Villalva R. P, Fontana F, Romo R. R,
Prieto R, et al. Gender Differences in the Acute Kidney Injury to Chronic Kidney Disease Transition. Sci Rep. 2017; 7: 12270 | DOI:10.1038/s41598-017-09630-2 1.

[20] Mosa O F, Fouad M A, Zafar T A, Fahmy A M, Alyazidi F and Rizk M. Epidemiology of acute kidney injury (aki) among hospitalized and outpatients frequent to al-lieth kidney unit (AKU). Epidemiology (Sunnyvale) 7: 317. doi:10.4172/21611165.1000317

[21] Bello BT, Busari AA, Amira CO, Raji YR, Braimoh RW. Acute kidney injury in Lagos: Pattern, outcomes, and predictors of inhospital mortality. Niger J Clin Pract 2017; 20:194-9.

[22] Murugan R, Kellum JA. Acute kidney injury: what's the prognosis. Nat Rev Nephrol 2011; 7:209-217.

[23] Yang L. Acute kidney injury in Asia. Kidney Dis (Basel) 2016; 2(3):95-102CrossRefGoogle Scholar.

[24] Thanachartwet V, Desakorn V, Sahassananda D, Kyaw Win KK, Supaporn T. Acute renal failure in patients with severe falciparum malaria: using the WHO 2006 and RIFLE criteria, Int J Nephrol, 2013, vol. 2013 pg. 841518.

[25] Franzen D, Rupprecht C, Hauri D, Bleisch JA, Staubli M, Puhan MA. Predicting outcomes in critically ill patients with acute kidney injury undergoing intermittent hemodialysis - A retrospective cohort analysis. Int J Artif Organs 2010;33:1521. 